\title{
Hsuan L. Hsu, Geography and the Production of Space in Nineteenth-Century American Literature
}

Cambridge, Cambridge University Press, 2010

\section{Julien Nègre}

\section{CpenEdition}

\section{Journals}

Édition électronique

URL : https://journals.openedition.org/transatlantica/6417

DOI : 10.4000/transatlantica.6417

ISSN : 1765-2766

Éditeur

Association française d'Etudes Américaines (AFEA)

Référence électronique

Julien Nègre, " Hsuan L. Hsu, Geography and the Production of Space in Nineteenth-Century American Literature », Transatlantica [En ligne], 1 | 2013, mis en ligne le 16 février 2014, consulté le 01 février 2023. URL : http://journals.openedition.org/transatlantica/6417 ; DOI : https://doi.org/10.4000/ transatlantica.6417

Ce document a été généré automatiquement le 1 février 2023

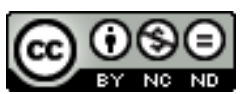

Creative Commons - Attribution - Pas d'Utilisation Commerciale - Pas de Modification 4.0 International - CC BY-NC-ND 4.0

https://creativecommons.org/licenses/by-nc-nd/4.0/ 


\title{
Hsuan L. Hsu, Geography and the Production of Space in Nineteenth- Century American Literature
}

\author{
Cambridge, Cambridge University Press, 2010
}

\author{
Julien Nègre
}

\section{RÉFÉRENCE}

HSU, Hsuan L., Geography and the Production of Space in Nineteenth-Century American Literature, Cambridge, Cambridge University Press, 2010, 257 pages, ISBN 0521197066, 70 euros.

Cet ouvrage de Hsuan L. Hsu s'intéresse à la façon dont la littérature américaine a assimilé les spectaculaires changements d'échelle qui ont marqué le XIX ${ }^{\mathrm{e}}$ siècle dans les domaines de la géographie, du commerce international, des migrations et de la colonisation. Fondant ses analyses sur les lectures marxistes de l'espace développées notamment par Henri Lefebvre et David Harvey, Hsu envisage ces changements d'échelle comme la conséquence de l'expansion spatiale du capitalisme qui, loin de créer un espace mondialisé homogène et uniforme, entraîne au contraire un « développement inégal » (uneven development) qui ne fait qu'accentuer les inégalités entre les espaces et les échelles différents. Les textes examinés par Hsu ont en commun de s'intéresser à des espaces spécifiques (frontière de l'expansion territoriale américaine, appartements urbains bourgeois, intimité du foyer domestique, îles du Pacifique, etc.) et de donner à voir les tensions qui y naissent de la confrontation entre l'échelle locale et l'échelle mondiale. Chaque chapitre envisage ainsi à la fois un « sousgenre majeur» (prominent subgenre, une notion problématique) et une situation géopolitique particulière. Le corpus de Hsu comprend des textes de Charles Brockden Brown, Edgar Allan Poe, Walt Whitman, Herman Melville, Catharine Beecher et Harriet 
Beecher Stowe, Henry James, l'écrivaine sino-américaine Sui Sin Far (Edith Eaton), Sarah Orne Jewett, Frank Norris, et Booker T. Washington.

2 L'ouvrage de Hsuan L. Hsu s'ouvre sur une description du célèbre et étrange tableau de Thomas Cole intitulé The Titan's Goblet (peint en 1833), qui représente une gigantesque coupe de pierre posée sur un paysage qui rappelle l'antiquité gréco-romaine. La coupe est remplie d'eau et des hommes semblent vivre sur les pourtours de ce lac artificiel entouré d'un cercle de forêt et totalement coupé du reste du monde. Au pied de la coupe, près de la côte, on distingue la silhouette d'un village ou d'une petite ville, et le tout baigne dans la lumière chaude du soleil en train de se coucher à l'arrière-plan.

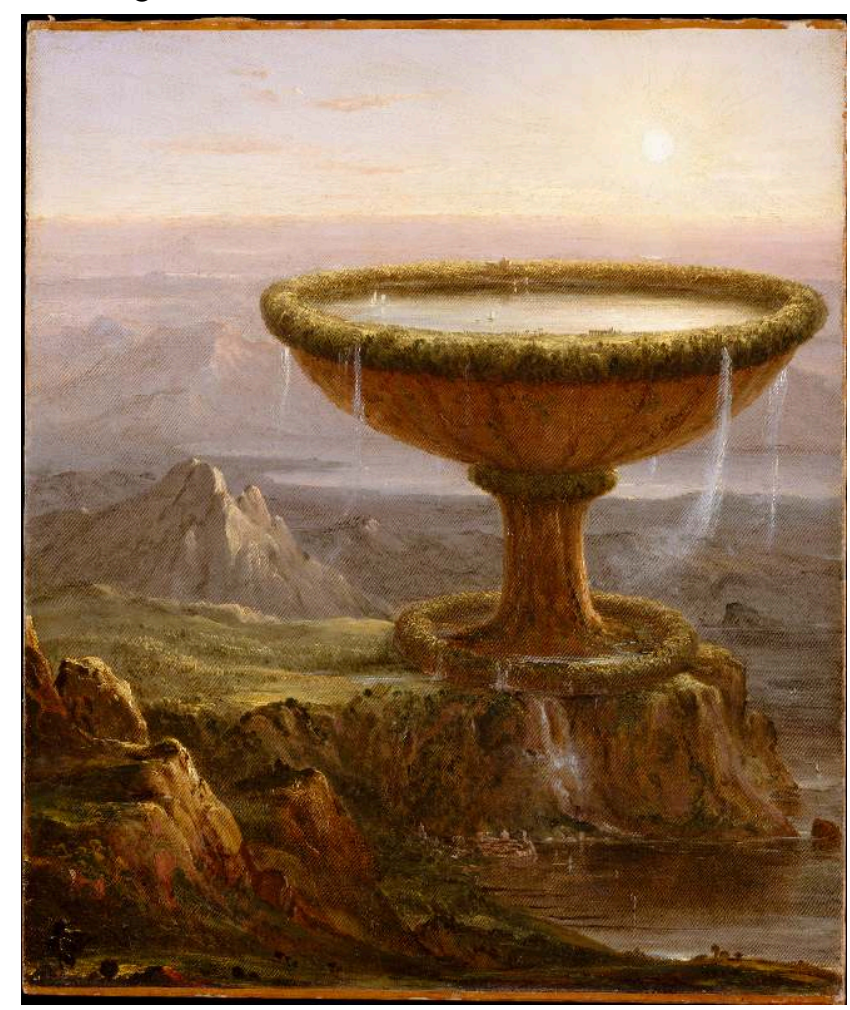

Thomas Cole, The Titan's Goblet, 1833, Huile sur toile, $49 \times 41 \mathrm{~cm}$, The Metropolitan Museum of Art, New York.

Source : Wikimedia Commons (http://upload. wikimedia.org/wikipedia/commons/0/05/ Cole\%2C_Thomas_-_Der_Pokal_des_Riesen_-_hi_res_-_1833.JPG).

Pour Hsu, ce tableau donne avant tout à voir une tension entre plusieurs échelles : celle de la nature morte (représentée par la coupe), celle de la polis classique et agrarienne et celle des espaces vastes, sublimes et sauvages. Ce télescopage d'échelles diverses est pour Hsu une caractéristique centrale du $\mathrm{XIX}^{\mathrm{e}}$ siècle et révèle une anxiété profonde liée à la brusque inclusion de l'espace local et familier dans un espace mondial que l'individu ne peut saisir mentalement. C'est cette tension et cette anxiété que Hsu explore dans une série de textes très variés qui vont des romans gothiques de Brown aux discours de Booker T. Washington sur le « New South» après la Reconstruction. Le plan de l'ouvrage est le suivant:

Introduction : scales of identification (1-24)

1. Democratic expansionism, Gothic geographies, and Charles Brockden Brown (25-56)

2. Urban apartments, global cities : the enlargement of private space in Poe and James (57-93)

3. Cultural Orphans : domesticity, missionaries, and China from Stowe to Sui Sin Far 
(94-128)

4. "The checkered globe" : cosmopolitan despair in the American Pacific (129-63)

5. Literature and regional production (164-95)

Epilogue : scales of resistance (196-207)

Le premier chapitre est consacré aux romans gothiques de Charles Brockden Brown et à la situation géopolitique particulière dans laquelle se trouvent les États-Unis au début du XIX siècle : ancienne colonie tout juste libérée du joug britannique, la jeune nation est en passe de devenir elle-même une puissance coloniale "impérialiste » en commençant à s'intéresser aux îles du Pacifique et en achetant la Louisiane à la France. Les textes de Brown examinés par Hsu sont notamment Wieland; or the Transformation (1798), son prequel inachevé Memoirs of Carwin the Biloquist, et Edgar Huntly; or, Memoirs of a Sleep-Walker (1799). Là où la grille du cadastre jeffersonien s'efforce de garder sous contrôle et d'uniformiser ces espaces nouveaux qui viennent s'ajouter au territoire national, les romans de Brown laissent entrevoir un malaise géographique qui fait bouger les lignes. Le ventriloquisme de Carwin, en faisant entendre une voix que l'on ne peut localiser dans l'espace et qui est comparée à une voix Mohawk, rend l'espace hétérogène, instable et impossible à cartographier. Il en va de même pour le somnambulisme dans Edgar Huntly, qui constitue une pratique aléatoire et inconsciente de l'espace. Pour Hsu, ces textes donnent ainsi à voir une attitude ambivalente face à l'expansion territoriale américaine : les îles du Pacifique sont envisagées comme le lieu possible d'une utopie démocratique, mais les rêves projetés en Océanie font ressortir par contraste les imperfections de la puissance coloniale sur son propre sol et les aspects sombres de la colonisation de l'Ouest, notamment en ce qui concerne le traitement des Amérindiens.

Le second chapitre s'intéresse à l'espace particulier que constitue l'appartement urbain bourgeois et à la façon dont il est envisagé dans les nouvelles policières de Poe (detective stories que Hsu appelle aussi detection stories puisque l'enjeu y est souvent d'arriver à voir ce qui est caché) mais aussi dans la description critique de l'Amérique moderne qu'offre Henry James dans The American Scene (1905-1906) et dans sa nouvelle «The Jolly Corner » (1908). En s'appuyant sur l'analyse des évolutions architecturales au cours du XIX ${ }^{\mathrm{e}}$ siècle, Hsu s'intéresse à la façon dont ces deux auteurs ont représenté l'espace clos et intime de l'appartement urbain. Pour Hsu, cet espace s'avère en réalité poreux et connecté à la circulation mondiale de marchandises et de migrants, comme l'illustre par exemple la présence inattendue du fameux orang-outan dans "The Murders in the Rue Morgue ». L'intersection entre l'espace privé de l'appartement et l'espace ouvert et public des échanges intérieurs se matérialise dans la fiction par la distorsion visuelle: vue "de côté » dans "The Murders in the Rue Morgue », objet caché aux yeux de tous dans "The Purloined Letter", perspective faussée dans "The Sphinx", etc. Les analyses de Hsu dans ce chapitre accordent ainsi une place importante à la vue et aux illusions d'optique. Quand James revient aux États-Unis après 25 ans d'absence et se trouve partagé entre la consternation devant une Amérique en pleine évolution, où l'immigration et les changements technologiques font apparaître une réalité différente, et une certaine forme d'enthousiasme devant ces changements innombrables, l'enjeu pour lui est de décider s'il doit remettre à jour ses propres «échelles» ou s'il doit conserver les anciennes pour pouvoir y mesurer la modernité. La nouvelle "The Jolly Corner » dramatise cette hésitation en mettant en scène un exilé qui revient au pays et se confronte à lui-même dans une grande maison bourgeoise vide au cœur de New York. 
6 Le troisième chapitre s'intéresse à l'espace du foyer familial et de la vie domestique (domesticity) mais en le mettant en rapport avec les entreprises missionnaires lancées par des Américain(e)s en Chine. Le corpus de Hsu comprend ainsi à la fois des textes d'écrivaines américaines sur la vie domestique, comme The American Woman's Home des sœurs Catharine Beecher et Harriet Beecher Stowe (1869), des textes écrits par des missionnaires américaines en Chine, et des textes écrits par des Sino-américaines qui ont connu l'expérience de la christianisation et de l'assimilation culturelle. Ce croisement de textes différents vise à apporter un éclairage nouveau sur ce qu'on a appelé « manifest domesticity » ou «tender violence », c'est-à-dire la double tendance des «fictions domestiques » américaines à, d'une part, décrire l'espace du foyer comme une sphère privée protégée du monde extérieur et, d'autre part, à chercher à étendre à l'extérieur ses valeurs raciales et religieuses, en une sorte d'impérialisme de la tendresse et de l'affection. En s'intéressant au cas particulier des enfants chinois ou d'origine chinoise dont les missionnaires américaines, en Chine ou en Californie, avaient fait leur cible, le chapitre de Hsu explore les dynamiques raciales et civilisationnelles qui structurent la perception de la domesticité et entreprend de montrer que ces textes sont souvent des idéalisations du travail missionnaire qui cherchent à occulter, sans y parvenir tout à fait, sa part de violence. Contrairement à l'image souvent développée, les enfants christianisés ne sont pas des "orphelins culturels " mais appartiennent à des familles qui leur ont transmis de fortes convictions religieuses et, une fois convertis, leur destin est bien souvent de devenir serviteurs dans les familles missionnaires. Les missionnaires américaines se doivent en effet d'offrir à la vue de tous un foyer exemplaire, mais le temps consacré au travail missionnaire les empêche d'assurer ces tâches domestiques elles-mêmes. Les serviteurs chinois deviennent alors des travailleurs subalternes indispensables au fonctionnement du système et, in fine, ils consolident l'idéal blanc d'une domesticité exemplaire. Ces textes se caractérisent ainsi par une attitude ambivalente vis-à-vis de l'altérité raciale, qu'il s'agit à la fois de convertir et assimiler, et de maintenir à distance dans une situation d'exploitation.

7 Le quatrième chapitre est consacré aux textes de Walt Whitman et Herman Melville qui évoquent le Pacifique, soit comme "passage » vers l'Asie, soit comme constellations d'îles exotiques : il s'agit bien sûr surtout de Typee (1846), Omoo (1847) Moby-Dick (1851) et «The Encatadas » mais aussi de poèmes tirés de John Marr (1888) et, pour Whitman, de poèmes comme «Salut au Monde!» et «Passage to India ». Pour Hsu, ces textes semblent implicitement valider l'expansion territoriale et l'accroissement des moyens de circulation autour du globe, mais ils mettent en réalité en scène une tension forte entre une mobilité internationale accrue et des moments d'immobilisation forcée et d'isolation individuelle (comme celui que connaît le narrateur de Typee, par exemple). Ces moments de " désespoir » (ce que Hsu appelle cosmopolitan despair) mettent ainsi en relief les contradictions et les aspects les plus sombres de la mondialisation et de la colonisation, c'est-à-dire, pour Hsu: "the uneven geographical development precipitated by imperialism and capital accumulation» (131). Au lieu de favoriser l'expansion de la démocratie à travers le monde, l'expansion territoriale américaine produit ce que Melville, dans John Marr, appelle " the checkered globe ", c'est-à-dire un patchwork de zones où le pouvoir, la richesse, la mobilité, la race et le travail se configurent de façons différentes. Pour Hsu, ces textes révèlent les contrastes entre ces zones, mais ils s'efforcent aussi d'envisager un moyen de les connecter, ne serait-ce que par des liens affectifs d'empathie et de solidarité. Or l'entreprise poétique touche ici à 
sa limite car la langue, pour pouvoir lier ces zones aux statuts différents et s'en emparer, ne peut qu'utiliser un langage « orbique " (l'expression vient de Whitman) homogène qui a pour conséquence de gommer les différences locales.

8 Le cinquième chapitre de cet ouvrage, pour finir, s'intéresse à l'espace spécifique que constitue la "région" américaine, qui est ici envisagée non pas comme une communauté affective fondée sur la nostalgie et le sentiment d'enracinement (rootedness) mais au contraire comme une unité spatiale définie par la production : à la fois en tant que région productrice d'un produit particulier et en tant qu'unité qui ne cesse d'être elle-même "produite " par les relations économiques et sociales qu'elle met en jeu et par la littérature qui l'évoque. Le chapitre décrit successivement trois régions et trois textes : le Maine producteur de bois de Sarah Orne Jewett, la Californie productrice de blé de The Octopus de Frank Norris et le Sud producteur de coton évoqué par Booker T. Washington. Dans chaque cas, et bien que le contexte et la portée de ces textes soient différents, Hsu explore comment la région en question est représentée comme une unité spatiale largement ouverte sur le monde et sur des réseaux d'échanges commerciaux, migratoires et économiques.

L'épilogue de l'ouvrage s'intéresse à une œuvre plus tardive: America Is in the Heart (1946) de l'écrivain philippin Carlos Bulosan, qui décrit notamment la situation des «Filipinos » immigrés aux États-Unis.

10 Les lectures que propose Hsu reposent sur des analyses textuelles détaillées souvent passionnantes et les œuvres sont systématiquement replacées dans leur contexte historique, c'est-à-dire les évolutions à la fois technologiques, sociales, géopolitiques et économiques qui ont façonné en profondeur ce réel que les textes évoquent. Isolément les unes des autres, ces analyses offrent de ce point de vue des lectures stimulantes et souvent originales des œuvres en question. Elles apportent dans chaque cas un éclairage particulier qui pourra être contrasté avec d'autres lectures.

11 Malgré tout, le propos d'ensemble de l'ouvrage n'est pas aisé à identifier. Pour commencer, la diversité du corpus, tout étant une des grandes richesses de cette étude, finit paradoxalement par le délégitimer : pourquoi s'intéresser à ces textes-là et pas à d'autres ? Certes, l'ensemble de ces textes donne à voir de manière frappante la façon dont l'élargissement spectaculaire de l'horizon géographique au $\mathrm{XIX}^{\mathrm{e}}$ a façonné les espaces les plus divers. Mais cela est-il vrai de tous les autres textes américains de cette période, ou seulement de ceux-ci ? La question est éludée. En qualifiant lui-même les différents types de textes qu'il étudie de prominent subgenres, Hsu ne prend pas le temps de préciser ce qui les définit, si ce n'est une attention à un type d'espace particulier. Le quasi-oxymore de l'expression " prominent subgenres » signale sa nature problématique : les textes qui sont étudiés ici sont-ils des sous-genres, c'est-à-dire des catégories mineures, produites à la marge d'autres genres plus canoniques, et qui tirent donc leur force de ce qu'elles s'opposent implicitement à une littérature dominante ? Ou sont-ils au contraire des variations localisées des genres dominants, qui reflètent donc les caractéristiques majeures de la plupart des textes de cette période? L'absence de certains auteurs chez qui les questions spatiales ont une importance particulière pousse aussi à s'interroger sur le choix du corpus : il n'est pas fait mention de James Fenimore Cooper, Mark Twain ni même de Henry David Thoreau, qui est sans doute le grand absent de ce panorama.

12 Par ailleurs, le choix d'un prisme de lecture unique limite sans doute l'ampleur des analyses possibles. Tout se passe comme si le capitalisme était systématiquement 
l'interlocuteur premier des textes évoqués ici, ce qui entraîne parfois une lecture manichéenne qui occulte d'autres dimensions de l'œuvre: pour dire les choses schématiquement, quand Ismaël s'enthousiasme pour les échanges planétaires et l'idée fascinante de la circumnavigation, il valide implicitement l'impérialisme américain et ses ravages. Dans la plupart des cas, les termes décrivant la mondialisation sont interchangeables avec "l'impérialisme américain » et «le capitalisme ». Les termes employés par Hsu pour décrire ce que ces textes « accomplissent » au juste vis-à-vis de ces tensions spatiales ne sont jamais très précis : «[they] make cognitive and emotional sense of the vast geographical transformations of their era» (1), "[they] mediate between individuals and different kinds of space» (2), «[they] explore» (163), etc. (je souligne). Cette imprécision tient bien sûr à la très grande variété des textes étudiés et à leur positionnement différent vis-à-vis des réalités socio-économiques qu'ils évoquent. Mais, pour cette raison-même, l'ouvrage de Hsu est davantage une série de lectures stimulantes et richement argumentées que la formulation d'une thèse unique sur la littérature américaine du XIX ${ }^{e}$ siècle.

\section{AUTEURS}

\section{JULIEN NÈGRE}

Université Paris-Diderot 